\title{
Repetition of deliberate self-harm and subsequent suicide risk: long-term follow-up study of II 583
}

\section{patients}

\author{
DANIEL LOUIS ZAHL and KEITH HAWTON
}

\begin{abstract}
Background Repetition of deliberate self-harm (DSH) is a risk factor for suicide. Little information is available on the risk for specific groups of people who deliberately harm themselves repeatedly.
\end{abstract}

\begin{abstract}
Aims To investigate the long-term risk of suicide associated with repetition of DSH by gender, age and frequency of repetition.
\end{abstract}

Method A mortality follow-up study to the year 2000 was conducted on II 583 people who presented to the general hospital in Oxford between 1978 and 1997. Repetition of DSH was determined from reported episodes prior to the index episode and episodes presenting to the same hospital during the follow-up period. Deaths were identified through national registers.

\section{Results Thirty-nine per cent of patients repeated the $\mathrm{DSH}$. They were at greater relative risk of suicide than the single- episode DSH group (2.24; 95\% Cl I.77- 2.84). The relative risk of suicide in the repeated DSH group compared with the single-episode DSH group was greater in females $(3.5 ; 95 \% \mathrm{CI}$ I.3-2.4) than males (I.8; 95\% Cl 2.3-5.3) and was inversely related to age (up to 54 years). Suicide risk increased further with multiple repeat episodes of DSH in females.}

\section{Conclusions Repetition of DSH is associated with an increased risk of suicide in males and females. Repetition may be a better indicator of risk in females, especially young females.}

Declaration of interest None. Funding detailed in Acknowledgements.
Deliberate self-harm (DSH) is often repeated (Sakinofsky, 2000). Repetition increases the risk of further DSH (Wilkinson \& Smeeton, 1987; Owens et al, 1994) and eventual suicide (Hawton \& Fagg, 1988; Tejedor et al, 1999). A less-studied aspect is the pattern of repetition over time and how this relates to suicide risk. Between 15 and $25 \%$ of people who deliberately harm themselves repeat the self-harm within 1 year of an index episode and present to the same hospital (Bancroft \& Marsack, 1977; Hawton et al, 1997; Owens et al, 2002). Few studies have examined repetition beyond 2 years of follow-up (Owens et al, 2002) and none has examined repetition over the long term. Little attention has been paid to how repetition influences risk in different age and gender subgroups. We have examined the short- and long-term risk of suicide in relation to repetition of DSH, including by gender, in a large sample of DSH patients, and also addressed the question of whether multiple repetition further increases the risk.

\section{METHOD}

\section{Study sample}

The sample was identified from the Oxford Monitoring System for Attempted Suicide. Through this system information is collected about each individual who presents to the general hospital in Oxford with an episode of DSH (Hawton et al, 1997; Hawton et al, 2003). The study cohort consisted of those patients who had presented with an episode of DSH between January 1978 and December 1997. Patients were grouped by age and gender at index episode (first recorded episode presenting to the general hospital in the study period). The age groups used were: $10-24,25-34,35-$ 54 and $>55$ years. Deliberate self-harm includes non-fatal self-poisoning and selfinjury. The former is defined as the intentional self-administration of more than the prescribed dose of any drug, whether or not there is evidence that the act was intended to cause self-harm. It also includes poisoning by non-ingestible substances and gas, provided that the hospital staff consider that these are cases of DSH. Alcohol intoxication is not included unless accompanied by other types of self-poisoning or self-injury. Self-injury is defined as any injury recognised by hospital staff as having been deliberately self-inflicted (Hawton et al, 1997).

\section{Repetition and mortality}

Patients were followed up until the end of December 2000; information regarding repetition and mortality was available for a potential maximum of 23 years.

Information on whether a patient had had an episode of DSH prior to the index episode was obtained through clinical interview. This was combined with data on episodes leading to referral to the same hospital during the follow-up period to allow all available data on repetition for each patient to be used, including the number of episodes and the distribution of episodes over time. For the purposes of this study, the repeated DSH group comprised patients who reported an episode of DSH prior to their index episode or who had a repeat episode of DSH during the followup period (or both). The single-episode DSH group comprised those with only an index episode of DSH. Those patients for whom there was no information on DSH history prior to the index episode were assumed to have no previous episodes.

Mortality status was determined by tracing the individual names and dates of birth against a national registry held by the Office for National Statistics (ONS) for England and Wales. The equivalent registries in Scotland and Northern Ireland were consulted for those found to have left the ONS register. Causes of death were obtained from death certificates supplied by the ONS.

Suicide was defined in line with current research practice (Adelstein \& Mardon, 1975; Charlton et al, 1992) based on coroners' verdicts of suicide (ICD code E950-E959), undetermined cause (E980E989) or accidental poisoning (E850E869). Patients who died from causes other than suicide, or who were alive at the end of the follow-up period, were included in a non-suicide group. 


\section{Statistical analyses}

All patients traced by the ONS for any length of time from their first presentation were entered into survival analyses. Kaplan-Meier curves were plotted and log-rank tests were used to test for differences in suicide risk between genders and age groups by DSH repetition status. Cox's regression models were fitted, testing for proportional hazards to estimate risk over time and according to gender and age at index episode.

The Kaplan-Meier curves shown in this paper are truncated at 15 years of followup time because numbers in some subgroups had fallen by then to $<20 \%$ of the original sample (Pocock et al, 2002). Analyses were carried out using SPSS version 10.0 for Windows.

\section{RESULTS}

\section{Patients and outcome}

A potential total of 12949 DSH patients was included in the investigation. Survival information was available on 11583 patients and these were included in the analysis. Analyses reported elsewhere showed no difference between the untraced and traced in terms of age, gender or method of DSH (Hawton et al, 2003). Patients were followed up for an average of 11.4 years (range 1 day to 23 years). Three hundred $(2.6 \%)$ had died by suicide and the remainder were either still alive at the end of the follow-up period (10394, $89.7 \%$ ) or had died from causes other than suicide $(889,7.7 \%)$.

At the time of the index episode, 2704 $(23.3 \%)$ patients reported at least one previous episode (1043, 22.6\% males; $1661,23.9 \%$ females). During the followup period, 2666 patients $(23.0 \%)$ repeated the DSH (1073, 23.2\% males; 1593, $22.9 \%$ females). By combining information about episodes prior to the index episode with data on repetition during the followup period, $4540(39.2 \%)$ were found to have repeated the DSH overall (1811, $39.2 \%$ males; 2729 , 39.2\% females). In total there were 18582 DSH episodes in the study period.

\section{Repetition and suicide risk}

Survival analysis showed that those who reported an episode of DSH prior to their index episode were at significantly greater risk of dying by suicide than those whose index episode was their first (log rank test:

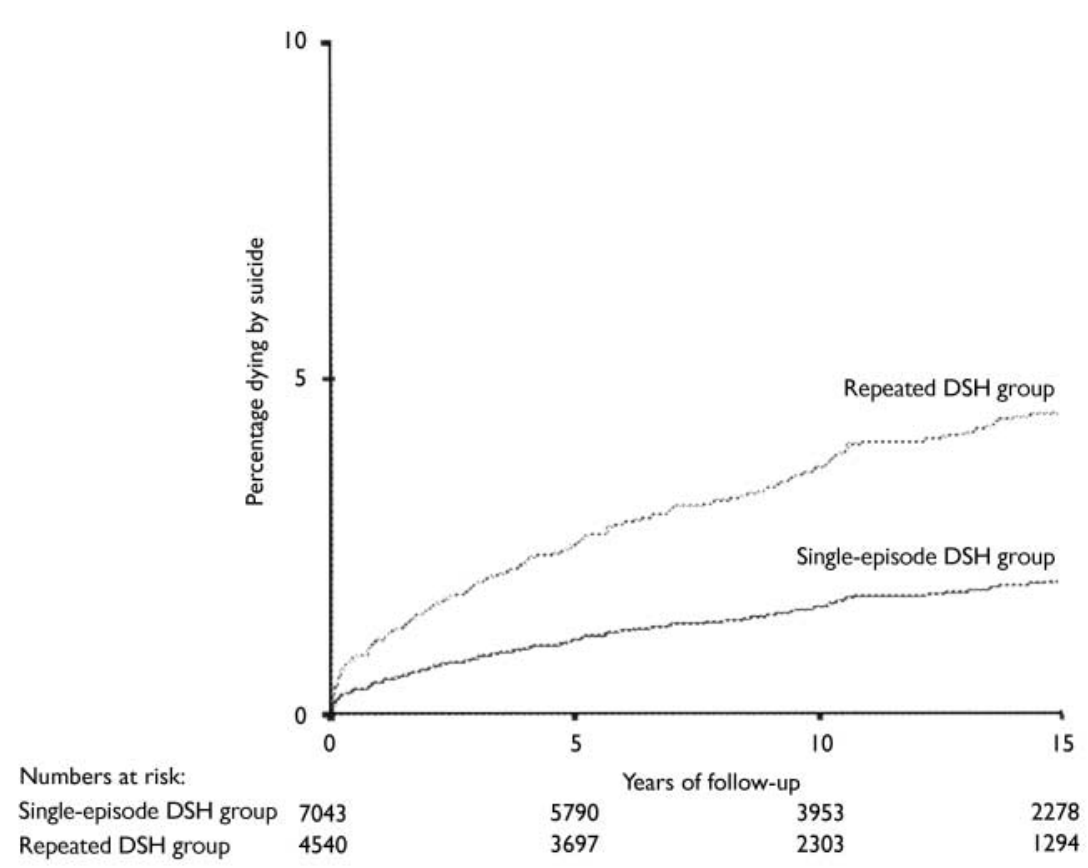

Fig. I Cumulative percentage of deaths by suicide in the study sample, by DSH repetition group.

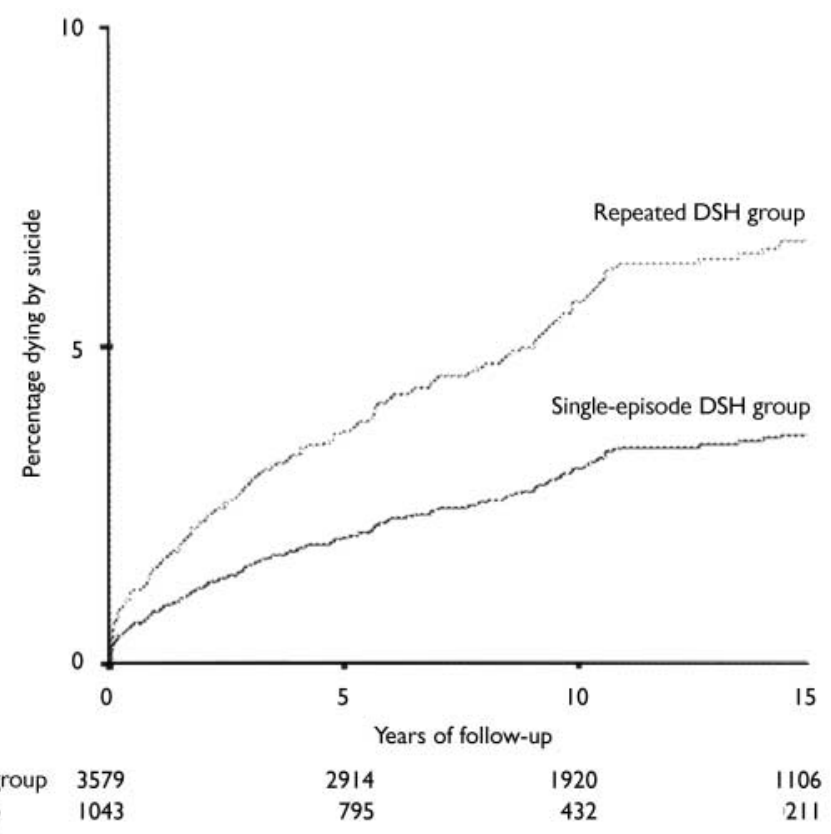

Fig. 2 Cumulative percentage of deaths by suicide among males in the study sample, by DSH repetition group.

$\left.\chi^{2}=27.06, P<0.001\right)$. Using our definition of repeated DSH (i.e. combining information on DSH repetition status at index episode with information about episodes during the follow-up period) showed a greater difference in risk between the repeated DSH group and the single-episode
DSH group (log rank test: $\chi^{2}=54.54$, $P<0.001$ ) (Fig. 1).

Survival analyses, using all data on repetition for the two genders separately, confirmed that those in the repeated DSH group were at significantly greater risk than those in the single-episode DSH group for 


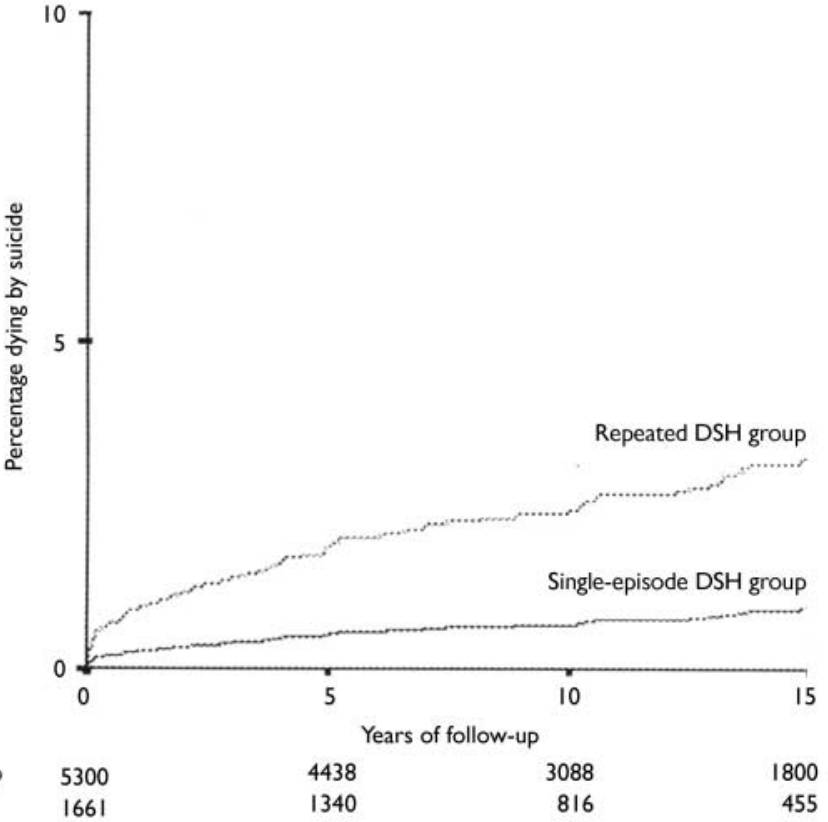

Fig. 3 Cumulative percentage of deaths by suicide among females in the study sample, by DSH repetition group.

both males (hazard ratio $1.88, P<0.001$, 95\% CI 1.42-2.50) and females (hazard ratio $3.58, P<0.001,95 \%$ CI $2.38-5.40$ ) (Figs 2 and 3 ).

The risk of suicide within 1 year of the index episode of DSH (Table 1 ) was $0.6 \%$ $95 \%$ CI $0.5-0.8)$ for the single-episode DSH group and $0.9 \%(95 \% \mathrm{CI}$ interval 0.6-1.2) for the repeated DSH group. The difference in risk between the two groups increased with time: after 15 years $1.9 \%$ (95\% CI $1.5-2.3)$ of the single-episode DSH group and $4.7 \%(95 \%$ CI $3.9-5.4)$ of the repeated DSH group had died by suicide (relative risk $=2.24 ; 95 \%$ CI 1.77 2.84).

Males in the repeated DSH group were at the greatest risk of suicide and females in the single-episode DSH group were at the least risk (Table 1). Within gender, the relative risk of suicide between the repeated DSH group and the single-episode DSH group over the study period was greater for females $(3.5$; $95 \%$ CI 2.3-5.3) than males $(1.8 ; 95 \%$ CI $1.3-2.4)$. The absolute risk for males regardless of repetition status was, however, higher than that of females in the repeated DSH group.

A comparison of risk of suicide according to repetition status and age group (Table 2) showed that up to the age of 54 years the ratio of risk of suicide in the repeated DSH group compared with the single-episode DSH group was inversely related to age. Thus, the ratio was particularly high in the youngest age group (10- to 24-year-olds). In this age group the relative risk of the repeated DSH group compared with the single-episode DSH group in males was 2.0 (95\% CI 1.2-3.3) and in females was 7.1 (95\% CI 2.9-17.3). Thus, young females in the repeated DSH group were at particularly increased risk of dying by suicide compared with those in the singleepisode DSH group. In fact, 25 (81\%) out of 31 young females who died by suicide were in the repeated DSH group.

\section{Multiple repetition}

All data on repetition were used to look at the comparative risk of suicide between those with more than two episodes including

Table I Risk of suicide (based on Kaplan-Meier estimates) after I, 5, 10 and I5 years of follow-up from index episode of deliberate self-harm (DSH), by repetition status and gender

\begin{tabular}{|c|c|c|c|c|c|c|c|c|c|c|c|c|c|}
\hline \multirow[t]{3}{*}{ Gender and repetition status } & \multirow[t]{3}{*}{$n^{\prime}$} & \multicolumn{12}{|c|}{ Time since first presentation for deliberate self-harm } \\
\hline & & \multicolumn{3}{|c|}{ I year } & \multicolumn{3}{|c|}{5 years } & \multicolumn{3}{|c|}{10 years } & \multicolumn{3}{|c|}{ I5 years } \\
\hline & & Suicides & $\begin{array}{c}\text { Risk } \\
\text { (\%) }\end{array}$ & $(95 \% \mathrm{Cl})$ & Suicides & $\begin{array}{c}\text { Risk } \\
\text { (\%) }\end{array}$ & $(95 \% \mathrm{Cl})$ & Suicides & $\begin{array}{c}\text { Risk } \\
\text { (\%) }\end{array}$ & $(95 \% \mathrm{Cl})$ & Suicides & $\begin{array}{c}\text { Risk } \\
\text { (\%) }\end{array}$ & $(95 \% \mathrm{Cl})$ \\
\hline \multicolumn{14}{|l|}{ Males } \\
\hline Single-episode DSH & 2811 & 22 & 1.0 & $(0.6-1.3)$ & 57 & 2.1 & $(1.6-2.6)$ & 77 & 3.4 & $(2.7-4.2)$ & 82 & 3.5 & $(2.7-4.3)$ \\
\hline Repeated DSH & 1811 & 27 & 1.2 & $(0.7-1.7)$ & 60 & 3.4 & $(2.6-4.3)$ & 84 & 5.5 & $(4.3-6.7)$ & 94 & 6.9 & $(5.5-8.4)$ \\
\hline Total & 4622 & 49 & I.I & $(0.8-1.4)$ & 117 & 2.6 & $(2.2-3.1)$ & 161 & 4.0 & $(3.5-4.7)$ & 176 & 4.8 & $(4.1-5.6)$ \\
\hline \multicolumn{14}{|l|}{ Females } \\
\hline Single-episode DSH & 4232 & 17 & 0.4 & $(0.2-0.6)$ & 25 & 0.6 & $(0.4-0.8)$ & 27 & 0.7 & $(0.4-0.9)$ & 31 & 0.9 & $(0.6-1.2)$ \\
\hline Repeated DSH & 2729 & 18 & 0.7 & $(0.3-1.0)$ & 45 & 1.7 & $(1.2-2.2)$ & 59 & 2.4 & $(1.8-3.1)$ & 69 & 3.3 & $(2.5-4.1)$ \\
\hline Total & 6961 & 35 & 0.5 & $(0.4-0.7)$ & 70 & 1.0 & $(0.8-1.3)$ & 86 & 1.4 & (I.I-I.7) & 100 & 1.8 & $(1.5-2.2)$ \\
\hline \multicolumn{14}{|l|}{ Both } \\
\hline Single-episode DSH & 7043 & 44 & 0.6 & $(0.5-0.8)$ & 82 & 1.2 & $(0.9-1.4)$ & 104 & 1.7 & $(1.3-2.0)$ & 113 & 1.9 & $(1.5-2.3)$ \\
\hline Repeated DSH & 4540 & 40 & 0.9 & $(0.6-1.2)$ & 105 & 2.4 & $(1.9-2.8)$ & 143 & 3.6 & $(3.0-4.2)$ & 163 & 4.7 & $(3.9-5.4)$ \\
\hline Total & II 583 & 84 & 0.7 & $(0.6-0.9)$ & 187 & 1.7 & (I.4-1.9) & 247 & 2.4 & $(2.1-2.7)$ & 276 & 3.0 & $(2.6-3.4)$ \\
\hline
\end{tabular}

I. $n=$ number of patients for whom there was any period of follow-up. 
Table 2 Proportions of patients who died by suicide over the entire follow-up period, by deliberate self-harm (DSH) repetition status, gender and age group

\begin{tabular}{|c|c|c|c|c|c|}
\hline & \multicolumn{5}{|c|}{ Age group (years) } \\
\hline & $10-24$ & $25-34$ & $35-54$ & $55+$ & All ages' \\
\hline & $\%$ (suicides $/ n$ ) & $\%$ (suicides/n) & $\%$ (suicides $/ n$ ) & $\%$ (suicides/n) & $\%$ (suicides $/ n$ ) \\
\hline \multicolumn{6}{|l|}{ Males } \\
\hline Single-episode DSH & $2.1(24 / 1157)$ & $3.2(25 / 792)$ & $5.2(32 / 619)$ & $3.3(8 / 24 I)$ & $3.2(89 / 28 I I)$ \\
\hline Repeated DSH & $4.1(31 / 758)$ & $5.8(33 / 566)$ & $7.0(27 / 387)$ & $12.0(12 / 100)$ & $5.7(103 / 1811)$ \\
\hline Relative risk $(95 \% \mathrm{Cl})$ & $2.0(1.2-3.3)$ & $1.8(1.1-2.9)$ & $1.4(0.9-2.2)$ & $3.6(1.5-8.6)$ & $1.8(1.4-2.4)$ \\
\hline \multicolumn{6}{|l|}{ Females } \\
\hline Single-episode DSH & $0.3(6 / 2208)$ & $0.6(5 / 830)$ & I.5 (I2/827) & $2.7(10 / 364)$ & $0.8(33 / 4232)$ \\
\hline Repeated DSH & 1.9 (25/I29I) & $2.5(16 / 631)$ & $3.3(20 / 607)$ & $7.0(14 / 200)$ & $2.7(75 / 2729)$ \\
\hline Relative risk $(95 \% \mathrm{Cl})$ & $7.1(2.9-17.3)$ & $4.2(1.6-11.4)$ & $2.3(1.1-4.6)$ & $2.6(1.2-5.7)$ & $3.5(2.7-4.7)$ \\
\hline \multicolumn{6}{|l|}{ Both } \\
\hline Single-episode DSH & $0.9(30 / 3365)$ & $1.8(30 / 1622)$ & $3.0(44 / 1446)$ & $3.0(18 / 605)$ & I.7 (I22/7043) \\
\hline Repeated DSH & $2.7(56 / 2049)$ & $4.1(49 / 1197)$ & $4.7(47 / 994)$ & $8.7(26 / 300)$ & $3.9(178 / 4540)$ \\
\hline Relative risk $(95 \% \mathrm{Cl})$ & $3.1(2.0-4.8)$ & $2.2(1.4-3.5)$ & $1.6(1.0-2.3)$ & $2.0(1.6-5.3)$ & $2.3(1.8-2.8)$ \\
\hline
\end{tabular}

I. Age not known for two males and three females.

Table 3 Risk of suicide for single and multiple repetition of deliberate self-harm (information on repetition based on overall study period)

\begin{tabular}{lcccc}
\hline Gender and type of repetition & $n$ & Suicides & Risk (\%) & $(95 \% \mathrm{Cl})$ \\
\hline Males & & & & \\
$\quad$ Single repetition & 1208 & 64 & 6.6 & $(4.8-8.4)$ \\
$\quad$ Multiple repetition & 603 & 39 & 7.5 & $(5.0-10.1)$ \\
Females & & & & \\
$\quad$ Single repetition & 1800 & 39 & 2.5 & $(1.7-3.3)$ \\
$\quad$ Multiple repetition & 929 & 36 & 4.7 & $(3.0-6.4)$ \\
Total & 4540 & 178 & 4.7 & $(3.9-5.4)$ \\
\hline
\end{tabular}

the index episode (multiple repetition) and two episodes only (single repetition). Thirty-four per cent (1532) of the repeated DSH group had more than two episodes (see Table 3). Overall, these patients were at an increased risk of death by suicide than were those with only two episodes of DSH over the follow-up period (log rank test: $\left.\chi^{2}=4.65, P=0.031\right)$. Survival analysis by gender revealed that females in the multiple repetition group were at an increased risk of suicide compared with the single repetition group ( $\log$ rank test: $\chi^{2}=5.98$, $P=0.015)$, but in males this was not so (log rank test: $\left.\chi^{2}=0.56, P=0.456\right)$.

\section{Survival time between last DSH episode and suicide}

The time between the date of the last recorded DSH episode during the study period and the date of suicide was compared for the repeated DSH group and the single-episode DSH group (Table 4). Those in the repeated DSH group had a shorter delay between the last DSH episode and suicide than did those in the single-episode DSH group (log rank test: $\left.\chi^{2}=47.55, P<0.001\right)$. Among the whole study sample, females survived for a shorter time than males following the last episode ( $\log$ rank test: $\left.\chi^{2}=19.45, P<0.001\right)$. Of those who died by suicide, males in the repeated DSH group survived for almost twice as long following their last episode as females in the repeated DSH group. Among those in the single-episode DSH group who died by suicide, males survived nearly three times as long as females.

\section{DISCUSSION}

In a previous report of this long-term follow-up study of a large sample of DSH patients we showed that risk of suicide following DSH was greater in males than in females and that it increased with age

Table 4 Median number of survival days between last episode of deliberate self-harm (DSH) and death from suicide by gender and repetition status (based on overall study period)

\begin{tabular}{|c|c|c|c|c|c|c|c|c|c|}
\hline & \multicolumn{3}{|c|}{ Males } & \multicolumn{3}{|c|}{ Females } & \multicolumn{3}{|c|}{ Both genders } \\
\hline & $n$ & Days & $(95 \% \mathrm{Cl})$ & $n$ & Days & $(95 \% \mathrm{Cl})$ & $n$ & Days & $(95 \% \mathrm{Cl})$ \\
\hline Single-episode DSH & 89 & 1038 & $(635-1457)$ & 33 & 348 & (39-796) & 122 & 891 & $(550-1139)$ \\
\hline Repeated DSH & 103 & 468 & $(306-750)$ & 75 & 268 & $(146-592)$ & 178 & 372 & $(275-383)$ \\
\hline Total & 192 & 741 & $(468-1058)$ & 108 & 289 & $(147-552)$ & 300 & 576 & $(37 I-770)$ \\
\hline
\end{tabular}


(Hawton et al, 2003). The present study is an in-depth investigation into the association between repetition of DSH and suicide in the same cohort of patients. It is based on a consistent approach to the monitoring of DSH (Hawton \& Fagg, 1992; Hawton et al, 1997, 2003).

\section{Methodological issues}

The number of repeat DSH episodes recorded will be an underestimate of the true number because only repeat episodes that resulted in presentation to the same general hospital as the index episode could be recorded. Episodes that presented to other hospitals and those that did not result in hospital presentation would not have been identified unless they occurred prior to the index episode.

Our method of identifying suicides by combining suicide verdicts with those of undetermined cause and accidental death due to poisoning was used because taking suicide verdicts alone underestimates the overall mortality from suicide (Charlton $e t$ $a l, 1992)$. The use of a national register to trace patients permitted an identification of outcome that was as accurate as possible because those who had changed names (especially females) and those who had moved away from Oxford during the follow-up period could still be traced. A drawback to the study is that it is based on patients presenting to only one general hospital. This hospital is, however, the main hospital for the catchment area. Also, the cohort size is very large compared with other studies. This permitted analyses by gender and age.

\section{Repetition of DSH}

The proportion of patients who had repeated the DSH at the time of the index episode $(23.3 \%)$ is well within the wide range that has been reported elsewhere (Sakinofsky, 2000; Owens et al, 2002). There was little difference in the proportions of males and females who had repeated the DSH at index presentation. There was also little difference between the proportions of males and females who repeated the DSH during the follow-up period, which is consistent with the findings of other studies (Morgan et al, 1976; Bancroft \& Marsack, 1977; Owens et al, 2002), although none has had access to such large cohorts.

\section{CLINICAL IMPLICATIONS}

Repetition of deliberate self-harm increases the risk of suicide over both the short and the long term, suggesting that specific suicide prevention strategies should be targeted at this group.

- The enhanced relative risk of suicide in females who deliberately harm themselves repeatedly compared with those with a single episode of deliberate self-harm suggests that repetition status as a risk factor may be particularly relevant when assessing risk in females.

- Assessment of repetition of deliberate self-harm should include enquiry about all episodes.

\section{LIMITATIONS}

- The study is based on patients presenting to only one general hospital.

- Repeat episodes after the index episode were recorded only if they presented to the same general hospital.

- Some patients could not be followed up.

DANIEL LOUIS ZAHL, BA, KEITH HAWTON, DSc, Centre for Suicide Research, University Department of Psychiatry, Warneford Hospital, Oxford, UK

Correspondence: Professor Keith Hawton, Centre for Suicide Research, University Department of Psychiatry,Warneford Hospital,Oxford OX3 7JX,UK. E-mail: keith.hawton@psych.ox.ac.uk

(First received 18 August 2003, final revision 12 January 2004, accepted 24 January 2004)

\section{Repetition and suicide risk}

The results confirm that repetition of DSH is associated with increased risk of eventual suicide (Hawton \& Fagg, 1988; Tejedor et al, 1999; Owens et al, 2002). The increased risk for those in the repeated DSH group in both the short and longer term compared with those in the single-episode DSH group suggests that detailed enquiry about previous DSH history is important in the assessment of risk. This should also include asking about episodes that have not resulted in presentation to hospital.

Although repetition of DSH may be one of the strongest risk factors for death by suicide (overall, it was confirmed in $59.9 \%$ of the suicides), it is also important to note that suicide occurred in a small minority of cases overall: $4.7 \%$ of those in the repeated DSH group died by suicide compared with $1.9 \%$ of those in the single-episode DSH group over 15 years. Attention to other risk factors (Sakinofsky, 2000 ) is also clearly necessary, although the difficulty in predicting the risk of suicide should not be underestimated (Hawton, 1987; Goldney, 2000).

This is the first study known to us that has looked at the risk of suicide according to repetition of DSH over such a long time period (15 years). It appears that repetition of DSH, especially when account is taken of repeat epsidoes during follow-up, is associated with continuing suicide risk, whereas for those in the single-episode DSH group there is a levelling-off of risk. Of course, further repeat episodes are likely to indicate ongoing or recurrent psychiatric and psychosocial problems, which may explain why risk persists. Nevertheless, the recurrence of actual self-harm is clearly a poor prognostic sign with regard to possible future suicide.

\section{Risk associated with repetition by gender, age and number of repeats}

Although male DSH patients have a greater overall risk of suicide than female patients (Hawton et al, 2003), the relative risk in 
the repeated DSH group compared with the single-episode DSH group is greater in females. Very young females in the repeated DSH group have a particularly high risk compared with those in the single-episode DSH group at the same-age. Thus, information about repetition may be more informative about future suicide risk in females than in males, and may be particularly useful in young females.

Because this is the only study we are aware of that has examined the risk of suicide in relation to repetition of DSH by both gender and age group, there is little previous work with which to compare our findings. However, our findings are consistent with the direction of the findings reported by Bancroft \& Marsack (1977).

Among all those in the repeated DSH group, those who engaged in multiple (more than two) episodes of DSH were at a significantly greater risk of suicide than those who repeated the DSH only once. The proportion of those with more than two episodes of DSH is consistent with that found elsewhere (Kreitman \& Casey, 1988). However, the increased risk in the multiple repetition group of the present study was almost entirely accounted for by females, which suggests that multiple repetition of DSH is a better predictor of risk in females than it is in males.

A shorter time was found between the last episode of DSH and death for those in the repeated DSH group compared with those in the single-episode DSH group. This may simply represent the greater risk in the repeated DSH group. However, the gender difference in time between the last DSH and suicide in both the repeated DSH group and the single-episode DSH group may indicate a gender difference in the persistence of risk of suicide behaviour. This requires further investigation.

\section{ACKNOWLEDGEMENTS}

This study was funded by a grant from the National Health Service Executive for England. K.H. is also funded by the Oxfordshire Mental Healthcare Trust. We thank the staff at the Office for National Statistics for England and Wales, the General Register Office for Scotland and the Central Services Agency in Northern Ireland for their assistance with this project. We also thank Louise Harriss for her advice on the statistical analyses. Staff at the Department of Psychological Medicine at the John Radcliffe Hospital in Oxford assisted with original identification of the patients included in the study.

\section{REFERENCES}

Adelstein, A. \& Mardon, C. (1975) Suicides 1961-74. Population Trends, 2, 13-19.

Bancroft, J. \& Marsack, P. (1977) The repetitiveness of self-poisoning and self-injury. British Journal of Psychiatry, I3I, 394-399.

Charlton, J., Kelly, S., Dunnell, K., et al (1992) Trends in suicide deaths in England and Wales. Population Trends, 69, $10-16$.

Hawton, K. (1987) Assessment of suicide risk. British Journal of Psychiatry, 150, 145-153.
Hawton, K. \& Fagg, J. (1988) Suicide, and other causes of death, following attempted suicide. British Journal of Psychiatry, I52, 359-366.

Hawton, K. \& Fagg, J. (1992) Trends in deliberate self poisoning and self injury in Oxford, 1976-90. BMJ, 304, |409-|4||.

Hawton, K., Fagg, J., Simkin, S., et al (1997) Trends in deliberate self-harm in Oxford, 1985-1995. Implications for clinical services and the prevention of suicide. British Journal of Psychiatry, I7I, 556-560.

Hawton, K., Zahl, D. \& Weatherall, R. (2003) Suicide following deliberate self-harm: long term follow-up study of patients who presented to a general hospital. British Journal of Psychiatry, 182, 537-542.

Kreitman, N. \& Casey, P. (1988) Repetition of parasuicide: an epidemiological and clinical study. British Journal of Psychiatry, 153, 792-800.

Morgan, H. G., Barton, J., Pottle, S., et al (1976)

Deliberate self-harm: a follow-up study of 279 patients. British Journal of Psychiatry, 128, 361-368.

Owens, D., Dennis, M., Read, S., et al (1994)

Outcome of deliberate self-poisoning. An examination of risk factors for repetition. British Journal of Psychiatry, 165, 797-80|

Owens, D., Horrocks, J. \& House, A. (2002) Fatal and non-fatal repetition of self-harm. Systematic review. British Journal of Psychiatry, 18I, 193-199.

Pocock, S., Clayton, T. \& Altman, D. (2002) Survival plots of time-to-event outcomes in clinical trials: good practice and pitfalls. Lancet, 359, 1686-1689.

Sakinofsky, I. (2000) Repetition of suicidal behaviour. In The International Handbook of Suicide and Attempted Suicide (eds K. Hawton \& K. Van Heeringen), pp. 385404. Chichester: John Wiley \& Sons.

Tejedor, M. C., Diaz, A., Castillon, J. J., et al (1999) Attempted suicide: repetition and survival - findings of a follow-up study. Acta Psychiatrica Scandinavica, 100, 205-2II.

Wilkinson, G. \& Smeeton, N. (1987) The repetition of parasuicide in Edinburgh 1980-1981. Social Psychiatry, 22, 14-19. 